\title{
Influencia de la combustión vehicular en la calidad del aire de Pamplona-Colombia
}

\author{
Alfonso Quijano Parra'; Mónica Juliana Quijano Vargas'; Iván Meléndez Gélvez² \\ ${ }^{1}$ Grupo de Investigación en Química. Laboratorio de Control de Calidad. Universidad de Pamplona. Colombia \\ ${ }^{2}$ Grupo de Investigación en Biología Molecular (BIOMOGEN).Universidad de Pamplona. Colombia
}

\section{Resumen}

La contaminación del aire por material particulado (PM) se considera un serio problema ambiental debido a la presencia en la atmósfera de materiales peligrosos tales como metales e hidrocarburos aromáticos poli cíclicos (HAP).

En los últimos tiempos ha tomado un gran auge en los países del tercer mundo incluida la América Latina, el estudio de la exposición humana a las emisiones de combustión provenientes de las fuentes móviles que circulan con gasolina y diésel que incluyen las partículas finas $\left(\mathrm{PM}_{2.5}\right)$ y los componentes muta génicos y carcinogénicos como son los HAP. Los HAP son uno de los contaminantes prioritarios del aire debido a sus propiedades extremadamente peligrosas para la salud humana. La materia orgánica presente en los filtros de $\mathrm{PM}_{2.5}$ del aire de Pamplona fue extraída con Diclorometano. Los efectos genotóxicos del material particulado $\mathrm{PM}_{2.5}$ del aire de Pamplona recolectado en una vía de alto tráfico vehicular por la cual circulan vehículos de carga pesada (tractomulas) que funcionan con diésel y vehículos livianos que funcionan con gasolina, fue evaluado mediante el ensayo cometa. Se identificó por cromatografía de gases (FID) un grupo de HAP considerados por la US EPA como contaminantes prioritarios; entre estos tenemos: naftaleno, fluoreno, fenantreno, pireno, benzo[a]antraceno y la mezcla indeno [1,2,3-cd] pireno, dibenzo[a,h]antraceno.

Palabras clave: ensayo cometa, genotoxicidad, Benzo(a) antraceno, cromatografía de gases, contaminación del aire.

\section{Influence of vehicular combustion air quality of Pamplona, Colombia}

\begin{abstract}
Air pollution by particulate matter (PM) is considered a serious environmental problem due to the presence in the atmosphere of hazardous materials such as metals and polycyclic aromatic hydrocarbons (PAHs).

In recent times it has taken a boom in third world countries including Latin America, the study of human exposure to combustion emissions from mobile sources circulating gasoline and diesel including fine particles (PM2. 5) and the mutagenic and carcinogenic components such as PAHs. PAHs are one of the priority air pollutants due to their extremely dangerous to human health properties. The organic matter in PM2.5 filters air Pamplona was extracted with diclorometano. The genotoxic effects of particulate matter PM2.5 air Pamplona collected in a high traffic route by which circulating heavy trucks that run on diesel and light vehicles that run


18

on gasoline, was evaluated by the comet assay. Was identified by gas chromatography (FID) a group of PAHs considered by the U.S. EPA as priority pollutants; among these are: naphthalene, fluorene, phenanthrene, pyrene, benzo[a]anthracene and the mixture Indene [1,2,3-cd] pyrene, dibenzo[a,h]anthracene.

Keywords: comet assay, genotoxicity, Benzo(a)anthracene, gas chromatography, air contamination.

*Para citar este artículo: Quijano Parra A; Quijano Vargas MJ; Melendez Gelvez I. Influencia de la combustión vehicular en la calidad del aire de Pamplona-Colombia. Revista Bistua.2016.14(1):17-31

+ Autor para el envió de correspondencia y la solicitud de las separatas: Alfonso Quijano Parra. Laboratorio de Control de Calidad. Grupo de Investigación en Química. Universidad de Pamplona. email: alfonsoquijanoparra@unipamplona.edu.co. 
19

\section{Introducción}

En los últimos años se están haciendo esfuerzos especiales con el fin de reducir la contaminación atmosférica y los efectos adversos que los contaminantes atmosféricos producen en la salud de la población. Algunos contaminantes como el dióxido de azufre $\left(\mathrm{SO}_{2}\right)$, óxidos de nitrógeno (NOx), monóxido de carbono (CO) y el material partículado (PM) que contiene hidrocarburos aromáticos poli cíclicos (HAP) y metales pesados que incluyen al plomo ${ }^{1}$ representan un grave riesgo para la salud humana ${ }^{2}$, se emiten a la atmósfera en grandes cantidades, provocando disminución de la calidad del aire ${ }^{3}$. Los tóxicos del aire son contaminantes que son conocidos 0 sospechosos de originar efectos adversos sobre el sistema inmunológico, el sistema nervioso e incluso originar cáncer ${ }^{4}$.

El material partìculado fracción respirable está dividido en dos grupos principales de acuerdo al tamaño de la partícula: $\mathrm{PM}_{10}$ y $\mathrm{PM}_{2.5}{ }^{5}$. Las partículas $\mathrm{PM}_{10}$ (diámetro aerodinámico $<10 \mu \mathrm{m}$ ) son partículas sólidas 0 líquidas dispersas en la atmósfera, en su mayoría de $\mathrm{pH}$ básico, conocidas como partículas finas, tienen la capacidad de penetrar y depositarse en la región traqueo bronquial del tracto respiratorio 6 . El segundo tamaño $\mathrm{PM}_{2.5}$ es un indicador de las partículas ultra finas (diámetro aerodinámico $<2.5 \mu \mathrm{m}$ ) agrupa partículas generalmente ácidas, estas partículas contienen hollín y otros derivados de las emisiones vehiculares $e$ industriales y corresponde a una fracción agresiva debido a que éstas son respirables ${ }^{7}$ y tienen mayor efecto en la salud, ya que pueden ingresar al sistema respiratorio depositándose en los alveolos pulmonares y llegar al torrente sanguíneo produciendo efectos negativos en la salud respiratoria en personas susceptibles ${ }^{8}$. En cuanto a los efectos sobre la salud humana, el PM fracción respirable es el de mayor importancia, ya que a largo plazo la exposición al PM se ha asociado con una mayor incidencia de enfermedades pulmonares, cardiovasculares y cáncer ${ }^{9}$. La masa orgánica extraíble de PM 10-2.5 emitidas de diversas fuentes de combustión (carbón, diesel y tabaco) induce tumores en los animales, mutaciones en las células; estudios epidemiológicos las han implicado claramente como carcinógenos humanos ${ }^{10}$. En los últimos tiempos en los países del tercer mundo incluida la América Latina, ha tomado auge el estudio de la exposición humana a las emisiones de combustión, incluyendo las partículas finas $\left(\mathrm{PM}_{2.5}\right)$ y los compuestos mutagénicos y carcinógenos como, los HAP y los nitro aromáticos policíclicos 11-14. Estudios de fraccionamiento del material particulado orgánico $\left(\mathrm{PM}_{2.5)}\right)$ del aire, han identificado HAP mutagénicos y carcinogénicos, que pueden causar daño oxidativo del ADN y pueden conducir a efectos cardiovasculares y reproductivos ${ }^{15}$. Se han realizado estudios para evaluar la población expuesta a HAP utilizando 
20

los datos de monitoreo ambiental 16-17. La identificación de mutágenos en partículas del escape de motores diésel y el aire urbano no sólo confirmó la contribución de los HAP a la actividad mutagénica, sino que también condujo al descubrimiento de nitroarenas altamente mutagénicas en las partículas del diésel del aire urbano ${ }^{18}$. En varios estudios se ha reportado que la contaminación del aire puede inducir en humanos cáncer, efectos respiratorios, daño genético, efectos reproductivos en ambos sexos y efectos cardiopulmonares 19-25. Una evaluación exhaustiva de la carcinogenicidad de la combustión de combustibles sólidos domiciliarios encontró evidencia de riesgo cancerígeno para los seres humanos 26 . Otro tipo de compuestos encontrados en las emisiones vehiculares son los hidrocarburos aromáticos sustituidos tales como nitroarenes (por ejemplo, nitro-PAH) y nitro-PAH lactonas, por ejemplo, lactonas nitropyrene, lactonas, nitrophenanthrene y 3nitrobenzanthrone) que se encuentran en el aire ambiente y en las partículas del diésel ${ }^{27}$. Uno de estos compuestos, 3-nitrobenzanthrone es un mutágeno inusualmente potente en el ensayo de mutagénesis bacteriana de Ames, recientemente ha sido reportado como inductor de tumores en roedores $28,29$. Una fuente importante de contaminación del aire en las zonas urbanas es la combustión de los combustibles diésel y gasolina usados en los automóviles, autobuses, camiones y otros 4 . Recientes estudios de distribución de las fuentes de la masa de partículas finas (PM 2.5) comprueban que los vehículos diésel y a gasolina son una de las principales fuentes de emisión de material particulado ${ }^{30}$. Los vehículos son también la principal fuente de partículas ultra finas cerca de las calles y carreteras 31-32. La 3nitrobenzanthrone (3-NBA) se aisló del diésel y de partículas de aire y ha demostrado ser un potente mutágeno de acción directa y también un carcinógeno en los humanos 27,28,33,35. Para la EPA la emisión del escape del diésel es probablemente cancerígena para los seres humanos por inhalación y concluye que existe un riesgo de cáncer a niveles de exposición ambiental 33. Según 36, las fuentes móviles a futuro contribuirán con un mayor riesgo de cáncer por la presencia de benceno, 1,3-butadieno, aldehídos, naftaleno, materia orgánica policíclica (MOP), además de partículas diésel y emisiones gaseosas orgánicas; por lo que la inhalación de sustancias tóxicas provenientes de las fuentes móviles seguirá siendo un problema de salud pública ${ }^{37}$. La intensidad del tráfico vehicular, influye en las características fisicoquímicas del PM, en particular con respecto a las concentraciones de HAP, metales y capacidad de generación de radicales 38,39. Los HAP suelen considerarse como un indicador de las emisiones del tráfico 40,41 y los HAP con 3-4 anillos, están asociados con las emisiones de los vehículos diésel ${ }^{40}$. Los HAP pueden 
21

crear toxicidad en organismos, al interferir con la función de la membrana celular y los sistemas de acoplamiento de enzimas, los metabolitos de HAP se pueden unir al ADN que causa interrupciones bioquímicas y daño celular a los organismos 42,43. La Agencia de Protección Ambiental de EE.UU. (US-EPA) recomienda el monitoreo de ciertos HAP conocidos como contaminantes prioritarios ${ }^{44,45}$, de gran preocupación debido a sus potenciales efectos cancerígenos y mutagénicos. Teniendo en cuenta el transporte vehicular entre las más relevantes fuentes de emisiones, esta investigación tiene como objetivo identificar los HAP presentes en el $\mathrm{PM}_{2.5}$ del aire de Pamplona y evaluar el riesgo para la salud mediante el ensayo genotóxico conocido como ensayo Cometa. El trabajo desarrollado incluyó la identificación de varios HAP considerados como prioritarios, mediante cromatografía de gases con detector FID, asociados con partículas finas $\mathrm{PM}_{2.5}$ recogidas en un sitio urbano de Pamplona-Colombia, influenciado por las emisiones de combustión del tráfico vehicular.

\section{Materiales y Métodos}

\section{Muestreo}

EL monitoreo del $\mathrm{PM}_{2.5}$ se realizó con un equipo Partisol Plus Model 2025-Air sampler. U.S.EPA. Reference designated $\mathrm{PM}_{2.5}$ Method RFPS 0498118 in accordance with 40CFR Part 53 de la Ruprecht-Patashnick. Se utilizaron filtros de Teflón de $47 \mathrm{~mm}$ de diámetro, con un tamaño de poro de 2 micras.

\section{Sitio de muestreo}

Se realizó el monitoreo de la fracción respirable ultra fina $\mathrm{PM}_{2.5}$ en Pamplona, Norte de Santander ubicada en la cordillera Oriental de Colombia con coordenadas geográficas $72^{\circ} 25$ de longitud Oeste y $7^{\circ} 20$ de latitud Norte, a una altitud de 2300 msnm y una presión atmosférica de $650 \mathrm{~mm}$ de $\mathrm{Hg}$; el equipo se instaló en la azotea del edificio de la Facultad de Ciencias Básicas de la Universidad de Pamplona ubicada en un sector residencial, cerca de una vía nacional por donde circula tráfico pesado (vehículos a motores diésel y gasolina) hacia la fronteriza ciudad de Cúcuta. Este sitio de monitoreo es muy importante, ya que en esta ciudad no existen fuentes externas de contaminación atmosférica y toda la contaminación atmosférica existente en este sitio es producida fundamentalmente por las fuentes móviles que circulan por esta vía. Las muestras ambientales obtenidas con el Partisol 2025 Plus en muestreos de 24 horas, cada tres días, se realizaron durante el período comprendido entre Enero-Julio del año 2012.

\section{Extracción de la Materia Orgánica de los filtros de $\mathbf{P M}_{2.5}$ de Pamplona.}

La materia orgánica de los filtros de $\mathrm{PM}_{2.5}$ (HAP) se extrae por ultrasonido en un baño ultrasónico (Branson 1510, modelo 1510R-MT); se utiliza como solvente de extracción el diclorometano grado cromatográfico suprasolv. Los filtros de $\mathrm{PM}_{2.5}$ provenientes del monitoreo diario se 
fracción se agregan $200 \mathrm{ml}$ de una

colocan en un vaso de precipitado con $20 \mathrm{~mL}$ del solvente por un periodo de 15 minutos a una temperatura de $23^{\circ} \mathrm{C}$ $24^{\circ} \mathrm{C}$ esta extracción se repite diez veces. Un procedimiento común para el análisis de los HAP consiste en la extracción seguido por el análisis instrumental, como la cromatografía de gases o la cromatografía líquida 46

\section{Concentración de la materia orgánica}

Una vez obtenido el extracto orgánico, se lo concentra en un rotaevaporador hasta aproximadamente $15 \mathrm{~mL}$ (extracto global). Posteriormente el extracto global se transfirió a tres viales cada uno de $5 \mathrm{ml}$, uno para la determinación de HAP por cromatografía de gases, otro para el fraccionamiento mediante columna de separación de silicagel y el último para los ensayos mutagénicos. Las muestras destinadas para la identificación de HAP se secaron con $\mathrm{Na}_{2} \mathrm{SO}_{4}$, con el fin de eliminar el agua residual y preparar la muestra para el análisis cromatográfico, se guardaron en frasco ámbar a $4^{\circ} \mathrm{C}$.

Fraccionamiento del extracto global de la materia orgánica del $\mathbf{P M}_{2.5}$

Se utiliza para el fraccionamiento del extracto global una columna de silicagel 47 , la cual tuvo un tratamiento térmico de ocho días a $170^{\circ} \mathrm{C}$ y durante dos días de $110^{\circ} \mathrm{C}$. Se coloca en una columna $10 \mathrm{~g}$ de silicagel, se agregan los $5 \mathrm{~mL}$ del extracto global al que se han adicionado $10 \mathrm{~mL}$ de Hexano. Posteriormente a esta columna se agregan $200 \mathrm{ml}$ de diclorometano, obteniéndose la fracción 1;obtenida esta mezcla diclorometano-hexano obteniéndose la fracción 2, posteriormente a la columna se agregan $200 \mathrm{~mL}$ de hexano obteniéndose la fracción 3 y por último se agregan $450 \mathrm{~mL}$ de metanol obteniéndose la fracción 4.

Identificación de Hidrocarburos Aromáticos Poli cíclicos (HAP)

Para identificar los hidrocarburos aromáticos policíclicos (HAP) presentes en el $\mathrm{PM}_{2.5}$ del aire de Pamplona (extracto global y las fracciones), se utilizó un equipo de cromatografía de gases marca Agilent Technologies 6890A Plus Series II Hewlet-Packard Plus con detector FID (Flame Ionization Detector). La columna utilizada es Restek Rxi-17 Sil MS, $30 \mathrm{~m}$ de longitud, $0.25 \mathrm{~mm}$ de diámetro, $0.25 \mu \mathrm{m}$ de diámetro interno (silarylene similar a $50 \%$ phenyl/50\% dimethyl polysiloxane). Para la identificación de los HAP se utilizó el patrón de 18 hidrocarburos de Restek (catalogo \# 31841 EPA Method 8310 $\mathrm{PAH}$ Mixture). La identificación cualitativa de los HAP presentes en el extracto global se realizó de acuerdo a las siguientes condiciones: detector FID a $320^{\circ} \mathrm{C}$ mezcla $(\mathrm{mL} / \mathrm{min})$ : aire 400 $-\mathrm{H}_{2} 30-\mathrm{N}_{2} 45$. Se inyectó $1 \mu \mathrm{l}$, modo splitless a $320^{\circ} \mathrm{C}$, temperatura inicial $65^{\circ} \mathrm{C}$ por $0,5 \mathrm{~min}$ y se incrementa de la siguiente manera: $15^{\circ} \mathrm{C} / \mathrm{min}$ hasta $200^{\circ} \mathrm{C}, 4^{\circ} \mathrm{C} / \mathrm{min}$ hasta $330^{\circ} \mathrm{C}$ durante $15^{\circ} \mathrm{C} / \mathrm{min}$, gas de arrastre helio, flujo $20 \mathrm{~mL} / \mathrm{min}$, temperatura del inyector $250^{\circ} \mathrm{C}$, tiempo de análisis por muestra $53,33 \mathrm{~min}$. 
$25 \mathrm{mM}$ y para el control negativo PBS.

Detección del daño en el ADN. Ensayo cometa

El ensayo cometa es una técnica altamente sensible para evaluar el daño y la reparación del ADN en cualquier tipo de célula eucariota. Este, en su versión alcalina, permite detectar roturas de cadena simple y sitios sensibles al álcali que se originan durante la reparación, dando lugar a la formación de la cola del cometa ${ }^{48}$. En general el principio básico del ensayo, es la migración del ADN en una matriz de agarosa bajo condiciones de electroforesis. Luego, al ser observada la célula al microscopio, presenta la apariencia de un cometa, con una cabeza (región nuclear) y cola (formada por fragmentos nucleares que han migrado en dirección del ánodo) por lo que este ensayo es también conocido como ensayo Cometa, debido al patrón de migración del ADN que se produce en las células dañadas.

\section{Extracción de linfocitos}

Se toman $5 \mathrm{~mL}$ de sangre total fresca de una persona sana y se mezcla suavemente con $5 \mathrm{~mL}$ de PBS. En otro tubo diferente se adicionan $3 \mathrm{~mL}$ de Histopaque y $9 \mathrm{~mL}$ de sangre, se centrifuga durante $30 \mathrm{~min}$ a $2.300 \mathrm{rpm}$ y se recoge la capa intermedia que es donde están los linfocitos

\section{Tratamiento}

A $200 \mu \mathrm{L}$ de células, se adiciona $50 \mu \mathrm{L}$ del tratamiento $O$ control (dosis $1=50$ $\mu \mathrm{g}$; dosis $2=100 \mu \mathrm{g}$; dosis $3=200 \mu \mathrm{g}$ ). Para el control positivo se utilizó $\mathrm{H}_{2} \mathrm{O}_{2}$
Posteriormente se incuban estas dosis y controles durante $1 \mathrm{~h}$ a $37^{\circ} \mathrm{C}$. Se toma $75 \mu \mathrm{L}$ agarosa de punto de fusión normal (LMA) y se mezcla con $10 \mu \mathrm{L}$ de células tratadas. Seguidamente la mezcla anterior se adiciona a la lámina base e inmediatamente se coloca el cubre objeto y se lleva a incubación durante $6 \min$ a $4^{\circ} \mathrm{C}$. Después de cumplidos los 6 min a $4^{\circ} \mathrm{C}$, se retira el cubre objeto y se adicionan otros $75 \mu \mathrm{L}$ de agarosa, se incuba durante $6 \mathrm{~min}$ a $4^{\circ} \mathrm{C}$. Terminado este tiempo se quita el cubre objeto y se incuba durante $1 \mathrm{~h}$ a $4^{\circ} \mathrm{C}$ en solución de lisis. A continuación se lavan las placas con PBS, luego se introducen en una cámara de electroforesis, después de 30 minutos se enciende la fuente de poder y se corre durante 30 minutos a 300 Amperios. Culminado el tiempo se procede a retirar de la cámara las placas; las cuales se lavan con solución neutralizante. Se dejan secar e inmediatamente se adiciona $30 \mu \mathrm{L}$ de bromuro de etidio. Luego se observa en el microscopio de fluorescencia Olympus U-RFKT50 con el objetivo de $25 \mathrm{X}$ y se mide la migración del ADN de 200 núcleos.

Análisis estadístico. Se determinó homogeneidad de varianzas usando la prueba de Levene. Si el comportamiento de los datos es paramétrico, se aplica Análisis de varianza (ANOVA). Si los datos son no paramétricos se utilizan las pruebas de Mann-Whitney y Wilcoxon. Se utilizó la prueba de Dunnett para determinar el nivel de significancia entre el 
Pamplona. Como se observa en este

tratamiento y control, así como la prueba de Tukey para comparaciones múltiples. Los valores se expresan como la media \pm la desviación estándar (X $\pm \mathrm{DS})$ y las pruebas se consideraron significativas con una $p \leq 0.05$.

Resultados y Discusión.

Identificación de hidrocarburos aromáticos policíclicos (HAP) por cromatografía de Gases/FID con la columna Restek RXI 17 Sil MX

Para la identificación de los diferentes HAP presentes en el extracto global del $\mathrm{PM}_{2.5}$ de Pamplona, se tomó como referencia el cromatograma de la muestra patrón de 18 hidrocarburos aromáticos policíclicos (EPA Method 8310 PAH Mix.), en este caso el cromatograma que se muestra en la Figura 1 se obtuvo con la columna Restek Rxi ${ }^{\circledR}-17$ Sil MS.

En la Figura 1 se muestra el cromatograma del patrón estándar de los 18 HAP (Restek EPA method 8310 PAH Mix), que corresponden al: 1 . naftaleno, 2. 1-metilnaftaleno, 3. -2metilnaftaleno, 4. acenaftileno, 5 . acenafteno, 6. fluoreno, 7. fenantreno, 8. antraceno, 9. fluoranteno, 10. pireno, 11. benzo(a)antraceno, 12. criseno, 13. benzo(b)fluoranteno, 14. benzo(k)fluoranteno, 15 . benzo (a) pireno, 16. indeno (1,2,3-cd) pireno, 17. dibenzo(a,h)antraceno, 18. benzo( $\mathrm{g}, \mathrm{h}, \mathrm{i})$ perileno.

En la Figura 2 se muestra el cromatograma correspondiente al extracto global de las muestras de la materia orgánica del PM 2.5 del aire de cromatograma los HAP identificados en el extracto global del aire de Pamplona son: Naftaleno, 1 metilnaftaleno, fluoreno, fenantreno y pireno. En la Figura 3 se muestra el perfil cromatográfico de la fracción 1. Los HAP identificados en la fracción 1 del extracto global del aire de Pamplona fueron: Naftaleno, Fluoreno, Fenantreno, Pireno y una mezca Indeno (1,2,3 -cd) pireno/ Dibenzo $(\mathrm{a}, \mathrm{h})$ antraceno.

Los HAP identificados en la fracción 2 (Figura 4) fueron: Naftaleno, 2 Metilnaftaleno, Fluoreno, Fenantreno, Pireno, Benzo(a) antraceno y la mezcla Indeno( 1,2,3 -cd) pireno/ Dibenzo $(\mathrm{a}, \mathrm{h})$ antraceno.

Los HAP identificados en la fracción 3 fueron: naftaleno, 2 metilnaftaleno, fluoreno, fenantreno, pireno y la mezcla Indeno( 1,2,3 -cd) pireno/ dibenzo $(\mathrm{a}, \mathrm{h})$ antraceno

Los HAP identificados en la fracción 4 fueron: fluoreno, pireno y la mezcla indeno $\quad(1,2,3-c d) \quad$ pireno/ dibenzo(a,h)antraceno.

Los resultados observados nos permiten deducir que el fraccionamiento de la materia organica del $\mathrm{PM}_{2.5}$, es importante en la determinación de los HAP, lo que permite identificar nuevos compuestos que no se detectan en el extracto global; en nuestro caso se identificó al Benzo(a)antraceno y la mezcla Indeno(1,2,3c-d)pireno/ Dibenzo(a,h) antraceno no encontrados en el

Bistua Revista de la Facultad de Ciencias Basicas .Universidad de Pamplona. ISSN 0120-4211 
25

extracto global y considerados por la IARC como posibles carcinógenos en humanos. En la Tabla 1 se muestran los HAP encontrados en el aire de Pamplona, extraídos con el diclorometano, tanto en el extracto global como en cada una de las cuatro fracciones

Tabla 1. HAP encontrados en el aire de Pamplona.

\begin{tabular}{|c|c|c|c|c|c|}
\hline Compuesto & $\begin{array}{c}\text { Mues. } \\
\text { Global }\end{array}$ & F1 & F2 & F3 & F4 \\
\hline Naftaleno & $\mathbf{X}$ & $\mathbf{X}$ & $\mathbf{X}$ & $\mathbf{X}$ & ND \\
\hline 1-Metilnaftaleno & $\mathbf{X}$ & ND & ND & ND & ND \\
\hline $\begin{array}{c}\text { 2-Metilnaftaleno } \\
\text { Fluoreno }\end{array}$ & $\mathbf{X D}$ & ND & $\mathbf{X}$ & $\mathbf{X}$ & ND \\
\hline $\begin{array}{c}\text { Fenantreno } \\
\text { Pireno }\end{array}$ & $\mathbf{X}$ & $\mathbf{X}$ & $\mathbf{X}$ & $\mathbf{X}$ & $\mathbf{N D}$ \\
\hline $\begin{array}{c}\text { Benzo(a)antraceno } \\
\text { d)pireno } \\
\begin{array}{c}\text { Dibenzo(a,h)antrace } \\
\text { no }\end{array}\end{array}$ & $\mathbf{X}$ & $\mathbf{X}$ & $\mathbf{X}$ & $\mathbf{X}$ & $\mathbf{X}$ \\
\hline $\begin{array}{c}\text { Indeno(1,2,3c- } \\
\text { detectado }\end{array}$ & $\mathbf{N D}$ & $\mathbf{X}$ & $\mathbf{X}$ & $\mathbf{X}$ & $\mathbf{X}$ \\
\hline
\end{tabular}

El dibenzo[a,h]antraceno, fue el primer HAP en demostrar tener capacidad carcinogénica. La Agencia de Protección Ambiental de los Estados Unidos (EPA) identifico los HAP más frecuentes y los clasificó como los 16 HAP prioritarios. Según este listado encontramos que el benzo[a]antraceno, indeno[1,2,3-cd]pireno se clasifican en el grupo 2B como posiblemente carcinogénico para humanos ;el Bistua Revista de la Facultad de Ciencias Basicas .Universidad de Pamplona. ISSN 0120-4211 dibenzo[a,h]antraceno se clasifica en el grupo 2A como probablemente carcinogénico para humanos. Es de anotar que estos HPA provienen exclusivamente de la combustión de las fuentes móviles que circulan con diésel y gasolina. Los resultados de los metales 49 e hidrocarburos aromáticos policíclicos (HAP) encontrados en el $\mathrm{PM}_{2.5}$ del aire de Pamplona son razonables, porque los orígenes de estos son principalmente emitidos de los tubos de escape del tráfico vehicular que es la principal fuente de $\mathrm{PM}_{2.5}$ en esta ciudad y concuerdan con un estudio realizado 50 en una zona de influencia netamente vehicular.

\section{Determinación del daño del ADN por ensayo cometa}

Se analizaron 200 células individuales (linfocitos) por cada dosis, con la finalidad de evaluar la fragmentación del ADN ocasionada por la exposición a contaminantes genotóxicos. Para llegar a ello, básicamente se tuvieron en cuenta los siguientes pasos: se obtuvieron las células (sangre total), posteriormente se fijaron con agarosa en un portaobjetos, las cuales fueron sometidas a una solución de lisis con la finalidad de romper su membrana celular, y además, se utilizó una solución amortiguadora para desenrollar el ADN, por la interrupción de los enlaces por puentes de hidrógeno entre las dobles cadenas del ADN, el paso a seguir, fue someter al ADN desenrollado a una electroforesis en tampón alcalino, en el cual los fragmentos negativamente cargados 
nos indica que existe un riesgo en la

de ADN (ADN dañado) migran fuera del núcleo en dirección al ánodo para formar un halo, apreciándose el daño, el cual es representado por un aumento de fragmentos del ADN que migran fuera de las células del núcleo bajo una forma característica similar a la cola de un cometa; estos fragmentos son generados por rompimientos del ADN; esto se puede observar por medio de un microscópico de fluorescencia; finalmente se utiliza un software para tabular estos datos y determinar el daño al material genético

Como control negativo se usó DMSO $12 \%$ que es el disolvente de las muestras de aire. El daño mostrado corresponde al cociente entre el daño producido por cada una de las dosis ensayadas con respecto al control negativo.

Todas las dosis analizadas inducen ruptura en el ADN de los linfocitos, se puede observar también que la dosis de $200 \mu \mathrm{g}$ de la fracción 3, induce un daño que supera 3 veces el control negativo, siendo estadísticamente significativo cuando se realizó el análisis de varianza. Como se puede observar, se está evidenciando daño en el ADN en cada una de las fracciones estudiadas en función de cada una de las dosis, lo que indica que en dichas fracciones existen compuestos responsables de ese daño. De acuerdo a los análisis por cromatografía de gases, podemos atribuir el daño genético a la presencia de compuestos tales como los HAP en el material particulado. Este resultado población expuesta, teniendo en cuenta que existe una correlación entre el incremento del daño en el ADN y cáncer. Estos hallazgos nos indican que parte de la genotoxicidad mostrada por el aire de Pamplona, es ocasionada por los HAP encontrados, dado que existe suficiente evidencia que correlaciona la presencia de estos compuestos y el riesgo para la salud humana.

\section{Conclusiones}

De los 8 HAP (HAP8) clasificados por la IARC como probables o posibles carcinógenos en humanos, se encontraron en el aire de Pamplona al benzo(a) antraceno y la mezcla indeno(1,2,3c-d) pireno y dibenzo(a,h) antraceno, considerados contaminantes altamente peligrosos por presentar actividad mutagénica y genotóxica.

Los ensayos cometa, realizados para determinar la genotoxicidad de las fracciones del $\mathrm{PM}_{2.5}$ del aire de Pamplona, mostraron que estas, ocasionan un daño en el material genético. Es probable que este daño sea ocasionado por los HAP encontrados en cada una de las fracciones de la materia orgánica extraída. 
27

\section{Referencias bibliogràficas}

1.-Muránszky G, Óvári M, Virág I, Csiba P, Dobai R, Záray G. Chemical characterization of PM10 fractions of urban aerosol. Microchem. J. 2011; 98:1-10

2.- Kunzli N, Kaiser R, Medina S. Publichealth impact of outdoor and trafficrelated air pollution: a European assessment. Lancet.2000; 356: 795801.

3.- European Environmental Agency (EEA), Annual European Community LRTAP Convention Emissions Inventory Report 1990-2006. EEA Technical Report No. 7, EEA, Copenhagen. 2008

4.- USEPA. 1990. Amendments to the Clean Air Act. U.S. Senate Committee on Environment and Public Works.2004.

5.- Samara C, Voutsa D. Size distribution of airborne particulate matter and associated heavy metals in the roadside environment. Chemosphere.2005; 59 (8):1197-1206.

6.- Vinitketkumnuen $U$, Kalayanamitra $K$, Chewonarin T, Kamens R. Particulate matter, PM10 \& PM2.5 levels, and airborne mutagenicity in Chiang Mai, Thailand. Mutat. Res.2002; 519:121-131

7.- Traversi D, Degan R, De Marco R, Gilli G, Pignata C, Villani S et al. Mutagenic properties of $\mathrm{PM}_{2.5}$ urban pollution in Northern Italy: the nitro- compounds contribution. Environ. Inter.2009; 35:905-910.

[8] Dominici F, Peng RD, Bell ML, Pham L, McDermott A, Zeger

Bistua Revista de la' Facultad de Ciencias Basicas .Universidad de Pamplona. ISSN 0120-4211
SL,et al. Fine particulate air pollution and hospital admission for cardiovascular and respiratory diseases. J. Am. Med. Assoc.2006; 295:1127-1134.

9.- Pope CA, Burnett RT,Thun MJ, Calle EE, Krewski D, Kazuhiko I, et al. Lung cancer, cardiopulmonary mortality,and long-term exposure to fine particulate air pollution,JAMA .2002;287:11321141.

10.- IARC. Diesel and gasoline engine exhausts and some nitroarenes, IARC Monographs on the Evaluation of the carcinogenic Risk of Chemicals to Humans, vol. 46, International Agency for Research on Cancer, Lyon, 1989.

11.- Claeys M, Graham B, Vas G, Wang W, Vermeylen R, Pashynska $V$ et al. Formation of secondary organic aerosol through photooxidation of isoprene. Science 2004; 303:1173-1176.

12.- Wu CF, Larson TV, Wu SY, Williamson J, Westberg $\mathrm{HH}$, Liu LJS. Source apportionment of PM2.5 and selected hazardous air pollutants in Seattle. Sci Total Environ. 2007; 386: $42-52$

13.-IARC.1984. Polynuclear aromatic compounds. Part 1. Chemical,environmental and experimental data, IARC Monographs on the Evaluation of Carcinogenic Risks to Humans, vol.32, International Agency for Research on Cancer, Lyon,1984 .

14.-IARC1984.Polynuclear aromatic compounds. Part 3. Industrial exposures in aluminum production, coal gasification, coke production, and iron and steel founding, IARC Monographs 
28

on the Evaluation of Carcinogenic Risks to Humans, vol. 34,International Agency for Research on Cancer, Lyon, 1984.

\section{5.-Lewtas J. Air pollution combustion} emissions: Characterization of causative agents and mechanisms associated with cancer, reproductive, and cardiovascular effects. Mut. Res. 2007; 636:95-133

16.- McCarthy MC, O'Brien TE, Charrier JG, Hather HR. Characterization of the chronic risk and hazard of hazardous air pollutants in the United States using ambient monitoring data. Environ Health Perspect. 2009; 117:790-796.

\section{7.- Wu CF, Wu SY, Wu YH, Cullen AC,} Larson TV, Williamson J, et al. Cancer risk assessment of selected hazardous air pollutants in Seattle. Environ Int . 2009; 35:516-522.

18.- Lewtas J. Genotoxicity of complex mixtures: strategies for the identification and comparative assessment of airborne mutagens and carcinogens from combustion sources. Fundam. Appl.Toxicol.1988; 10:571-589.

\section{9.- Samet JM. Environmental causes of} lung cancer: what do we know in 2003? Chest .2004; 125:80S-83S.

20.- Næss O, Nafstad P, Aamodt G, Claussen B, Rosland P. Relation between concentration of air pollution and cause specific mortality: four-year exposures to nitrogen dioxide and particulate matter pollutants in 470 neighborhoods in Oslo,Norway. Am. J. Epidemiol.2007; 165 (4):435-443.

21.- Horstman D, Kotesovec F, Nozicka children in highly polluted Northern Bohemia. Arch. Environ. Health.1997; 52: 56-62

22.-inkova B, Lewtas J, Miskova I, Lenicek J, Sram R. DNA adducts and personal air monitoring of carcinogenic polycyclic aromatic hydrocarbons in an environmentally exposed population. Carcinogenesis. 1995; 16:1037-1046.

23.- Binkova B, Lewtas J, Miskova I, Rossner P, Cerna M, Mrackova G,et al. Biomarkers studies in the Northern Bohemia, Environ. Health Perspect.1996; 104: 591-597

24.- Selevan SG, Borkovec L, Slott VL, Zudova Z, Rubes J, Evenson DP,et al. Semen quality and reproductive health of young Czech men exposed to seasonal air pollution. Environ. Health Perspect.2000; 108:887-894.

25.-Peters J, Skorkovsky F, Kotesovec J, Brynda C, Spix HE, Wicehmann J,et al. Associations between mortality and air pollution in Central Europe, Environ. Health Perspect.2000; 108:283-287.

26.- Straif K, Baan R, Grosse $Y$, Secretan B, Ghissassi F, Cogliano V. Carcinogenicity of household solid fuel combustion and of high-temperature frying, WHO International Agency for Research on Cancer Monograph Working Group, Lancet Oncol.2006; 7 :977-978 (Summary of IARC Monograph, vol. 95).

27.- Enya $T$, Suzuki $H$, Watanabe $T$, Hirayama T, Hisamatsu $Y$. 3Nitrobenzanthrone a powerful bacterial mutagen and suspected human carcinogen found in diesel exhaust and 
29

airborne particles. Environ. Sci. Technol.1997; 31:2772-2776.

28.- Arlt VM. 3-Nitrobenzanthrone, a potential human cancer hazard in diesel exhaust and urban air pollution: a review of the evidence. Mutagenesis.2005; 20(6): 399-410.

29.- Nagy E, Zeisig M, Kawamura K, Hisamatsu Y, Sugeta A, Adachi S,et al. DNA adduct and tumor formations in rats after intratracheal administration of the urban air pollutant 3-nitrobenzanthrone. Carcinogenesis.2005; 26(10):1821-1828.

30.- Maykut NN, Lewtas J, Kim E, Larson TV. Source apportionment of PM2.5 at an urban improve site in Seattle, WA, Environ. Sci. Technol.2003; 37:5135-5142

31.- Palmgren F, Wahlin P, Kildeso J, Afshari A, Fogh C. Characterisation of particle emissions from the driving care fleet and the contribution to ambient and indoor particle concentrations, Phys. Chem. Earth.2003; 28:327-334.

32.-Lewtas J, Huisingh R, Bradow R, Jungers $R$, Claxton L, Zweidinger $R$. Application of bioassay to the characterization of diesel particle emissions, in: M.D.Waters, S. Nesnow, J.

LewtasHuisingh, et al. (Eds.), Application of Short-term Bioassays in the Fractionation and Analysis of Complex Environmental Mixtures, Plenum Press, New York. 1979: 383-418.

33.- US-EPA. Health Assessment Document for Diesel Engine Exhaust. US Environmental Protection Agency. Office of Research and Development. National Center for Environmental Assessment. Washington, DC, 2002 (EPA/600/8 Bistua Revista de la Facultad de Ciencias Basicas .Universidad de Pamplona. ISSN 0120-4211
90/057F)[34] Batía R, Lopipero P, Smith A. Diesel exhaust exposure and lung cancer, Epidemiol.1998; 9 (1):84-91

35.- Lipsett M, Campleman S. Occupational exposure to diesel exhaust and lung cancer: a meta-analysis. Am. J. Public Health.1999; 80 (7):1009-1017.

36.- Cook R, Strum M, Touma JS, Palma T,Thurman J, Ensley D,et al. Inhalation exposure and risk from mobile source air toxics in future years, J. Exp. Sci. Environ. Epidemiol.2007; 17:95-105.

37.- Parsons B, Salter LF.Air quality effects of traffic in a canyon like street (Falmouth, U.K.). Environ. Monit.

Assess.2003: 82: 63-73.

38.- Vera Castellano A, Lopez Cancio J, Santana Aleman P, Santana Rodriguez J. Polycyclic aromatic hydrocarbons in ambient air particles in the city of Las Palmas de Gran Canaria. Environ. Int.2003; 29: 475-480.

39.- Guo H, Lee SC, Ho KF, Wang XM, Zou SC. Particle-associated polycyclic aromatic hydrocarbons in urban air of Hong Kong, Atmos.

Environ.2003;37:5307-5317.

40.- Vardar N, Esen F, Tasdemir Y. Seasonal concentrations and partitioning of PAHs in a suburban site of Bursa, Turkey. Environ. Pollut. 2008; 155:298 307.

41.- Kap-Soon N, Do-Youn L, Cha JH, Joo WA, Lee E, Chan-Wha K. Protein biomarkers in the plasma of workers occupationally exposed to polycyclic aromatic hydrocarbons. Proteomics. 2004; 4:3505-3513. 
30

42.- Gozgit JM, Nestor KM, Fasco MJ, Pentecost BT, Arcaro KF. Differential action of polycyclic aromatic hydrocarbons on endogenous estrogen-responsive genes and on a transfected estrogen-responsive reporter inMCF-7cells.Toxicol. Appl. Pharmacol. 2004; 196:58-67.

43.- Kosmehl T, Hallare AV, Braunbeck T, Hollert $\mathrm{H}$. DNA damage induced by genotoxicants in zebrafish (Danio rerio) embryos after contact exposure to freeze dried sediment and sediment extracts from Laguna Lake (The Philippines) as measured by the comet assay. Mutat. Res.2008; 650:1-14.

44.- Shibamoto T. Chromatographic Analysis of Environmental and Food Toxicants. Marcel Dekker, INC. New York.1998

45.- United States Environmental Protection Agency (US-EPA). Guidelines for Carcinogen Risk Assessment. Federal Register.1986, 51(185), pp. 33992 34003.EPA/630/R-00/004,Washington, DC.

46.- Ping L, Pennwalt H. Characterization of polycyclic aromatic hydrocarbons (PAHs) on lime spray dryer (LSD) ash using different extraction methods.

Chemosphere.2006; 62:265-274.

47.- Xiao-Yang $\mathrm{Y}$, Igarashi $\mathrm{K}$, Tang $\mathrm{N}$, Lin $\mathrm{JM}$. Indirect- and direct-acting mutagenicity of diesel, coal and wood burning-derived particulates and contribution of polycyclic aromatic hydrocarbons and nitropolycyclic aromatic hydrocarbons. Research. 2010; 695:29-34. Academy of Environmental Sciences, Beijing, China.
Temperatura sobre la Reparación del Daño Genético inducido por Peróxido de Hidrógeno y la Radiación Ultravioleta A en Células Sanguíneas Humanas. Acta Farm. Bonaerense.2004; 23(3):277-84.[49]

49.-Quijano Parra A, Quijano Vargas MJ, Henao M JA. Caracterización fisicoquímica del Material Particulado-Fracción Respirable (PM2.5) en Pamplona (Colombia). Bistua: Revista de la Facultad de Ciencias Básicas.2010; 10(1):1-11.

50.- Meléndez Gélvez I, Martínez Montañez ML, Quijano Parra.A. Actividad mutagénica y genotóxica en el material particulado fracción respirable $\mathrm{MP}_{2,5}$ en Pamplona, Norte de Santander, Colombia. latreia .2012; 25 (4): 347-356

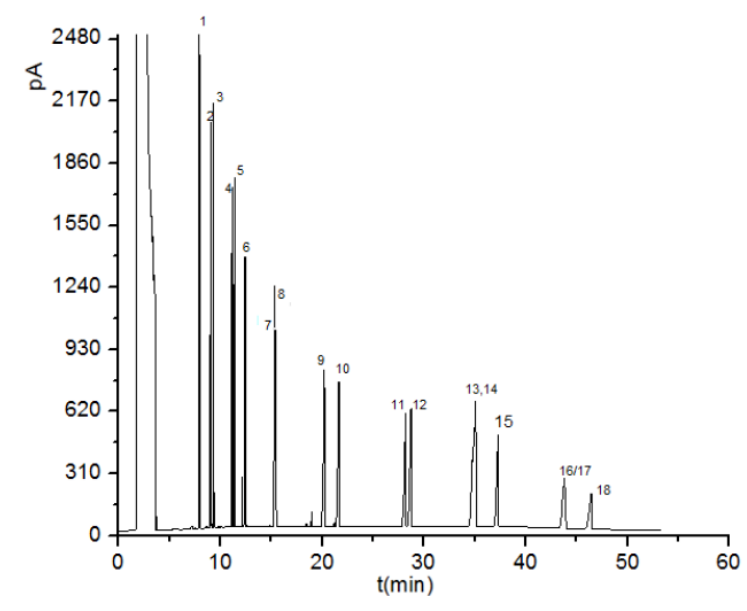

Figura 1. Cromatograma del patrón estándar de los 18 HAPs (Restek EPA method 8310 PAH Mix.

48.- Ayala Mayte Cossio. Uso del Ensayo

Cometa para Evaluar el Efecto de la

Bistua Revista de la Facultad de Ciencias Basicas .Universidad de Pamplona. ISSN 0120-4211 

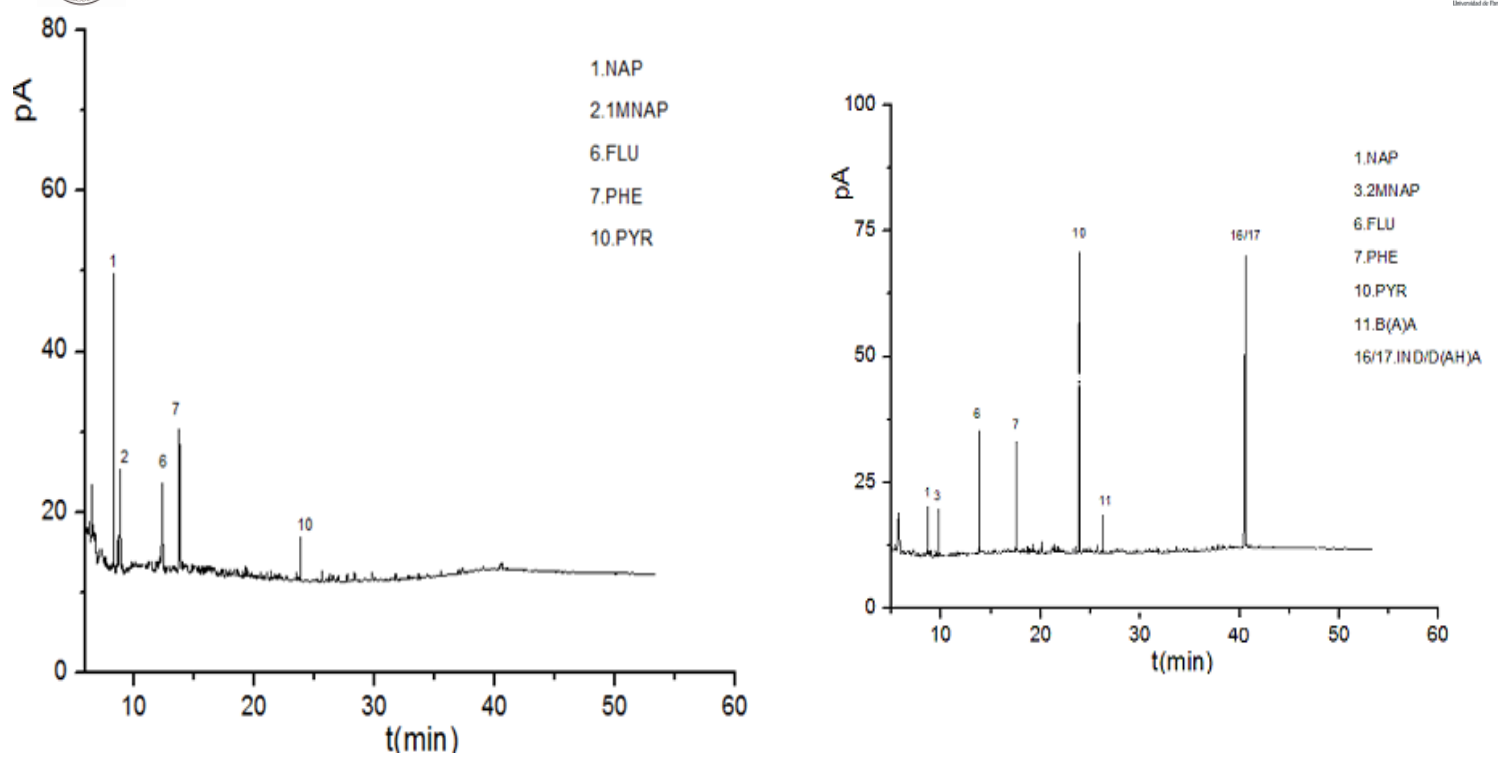

Figura 2. cromatograma correspondiente al extracto global de las muestras de la materia orgánica del $\mathrm{PM}_{2.5}$ del aire de Pamplona.

Figura 3. Perfil cromatografico de la fracción 1.

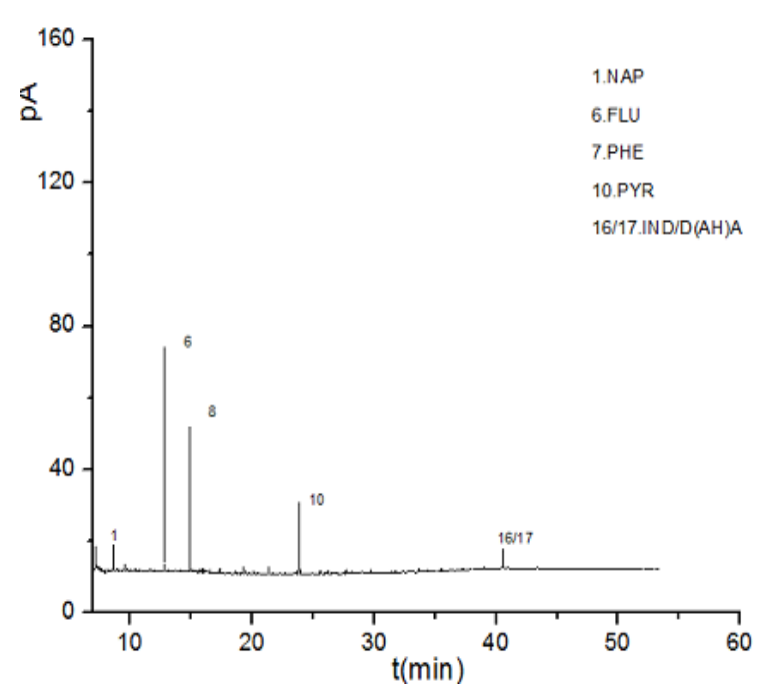

Figura 4. Perfil cromatografico de la fracción 2.
Alfonso Quijano Parra. Ph.D. Investigador Asociado. Director laboratorio Control de Calidad Universidad de Pamplona. Director Grupo de Investigación en Química.

Mónica Juliana Quijano Vargas.

Especialista en Bioquímica. Grupo de Investigación en Química. Universidad de Pamplona.

Iván Meléndez Gélvez. Ph.D.

Investigador Asociado.Director. Director Grupo de Investigación en Biología Molecular. Universidad de Pamplona 